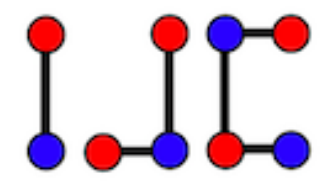

\title{
The locating-chromatic number and partition dimension of fibonacene graphs
}

\author{
Ratih Suryaningsih, Edy Tri Baskoro \\ Combinatorial Mathematics Research Group, \\ Faculty of Mathematics and Natural Sciences, Institut Teknologi Bandung, \\ Jalan Ganesa 10 Bandung, Indonesia \\ ratihsuryaningsih09@gmail.com, ebaskoro@math.itb.ac.id
}

\begin{abstract}
Fibonacenes are unbranched catacondensed benzenoid hydrocarbons in which all the non-terminal hexagons are angularly annelated. A hexagon is said to be angularly annelated if the hexagon is adjacent to exactly two other hexagons and possesses two adjacent vertices of degree 2 . Fibonacenes possess remarkable properties related with Fibonacci numbers. Various graph properties of fibonacenes have been extensively studied, such as their saturation numbers, independence numbers and Wiener indices.

In this paper, we show that the locating-chromatic number of any fibonacene graph is 4 and the partition dimension of such a graph is 3 .

Keywords: fibonacenes, locating-chromatic number, partition dimension

Mathematics Subject Classification: 05C15

DOI: $10.19184 /$ ijc.2019.3.2.5

\section{Introduction}

Many applications of graphs can be found in chemistry. Chemical graphs were first introduced in the middle of the 18th century. Graph theory is increasingly popular in chemistry because almost all problems in chemistry can be modeled in simple graphs that directly represent molecules, chemical bonds and so on. Furthermore, graph theory provides simple rules so that it can obtain many qualitative predictions about the structure and reactivity of various compounds. Seeing the benefits of graph theory in chemistry, this is interesting to discuss.
\end{abstract}

Received: 8 Jul 2019, Revised: 27 Sep 2019, Accepted: 16 Dec 2019. 
Gutman dan Klavžar [12] discussed the chemical graph theory of fibonacenes. A benzenoid system is a connected plane graph without cut-vertices in which all inner faces are hexagons, such that two hexagons are either disjoint or have exactly one common edge, and no three hexagons share a common edge. A benzenoid system is said to be unbranched catacondensed if it does not possess a vertex shared by three hexagons and not possess a hexagon having three neighboring hexagons. Fibonacenes are unbranched catacondensed benzenoid hydrocarbons in which all the non-terminal hexagons are angularly annelated. A hexagon is said to be angularly annelated if the hexagon is adjacent to exactly two other hexagons and possesses two adjacent vertices of degree 2. Previously fibonacenes were introduced by Balaban [5], although it seems to be first mentioned by Anderson [2]. Fibonacenes have the Kekulé structure, which is in graph theory it is called a perfect matching. The number of perfect matching from fibonacenes with $h$ hexagon forms Fibonacci numbers. Balaban [5] gives the name fibonaccene. However, he suppressed one $c$ for simplicity and for similarity with the established name "acenes".

There are several papers that have discussed benzenoid specifically fibonacenes, such as Wiener index [9], saturation number [10], and independence number [15]. The applications of these topics in chemistry have also been mentioned in $[9,10,15]$.

The concept of the locating-chromatic number was first introduced by Chartrand et al. [6]. The locating-chromatic number concept of a graph is as a combination of two concepts that is the graph coloring concept and the partition dimension concept. The concept of the partition dimension of a graph was introduced by Chartrand et al. [7] as an extension of the metric dimension concept introduced by Harary and Melter [13].

In this paper, we consider only simple connected graphs. Let $G(V, E)$ be a graph with the vertex-set $V$ and edge-set $E$. For a vertex $v$ of a connected graph $G$ and a subset $S$ of $V(G)$, the distance between $v$ and $S$ is $d(v, S)=\min \{d(v, x) \mid x \in S\}$. Let $N(v)$ be the set of vertices adjacent to $v$. For an ordered $k$-partition $\Pi=\left\{S_{1}, S_{2}, \ldots, S_{k}\right\}$ of $V(G)$, the representation of $v$ with respect to $\Pi$ is the ordered $k$-tuple $r(v \mid \Pi)=\left(d\left(v, S_{1}\right), d\left(v, S_{2}\right), \ldots, d\left(v, S_{k}\right)\right)$. The $k$ partition $\Pi$ is a resolving partition if the ordered $k$-tuple $r(v \mid \Pi), v \in V(G)$, are distinct. The minimum $k$ for which there is a resolving $k$-partition of $V(G)$ is the partition dimension $p d(G)$ of $G$.

Chartrand et al [8] characterized all graphs with partition dimension 2, and all graphs on $n$ vertices with partition dimension $n-1$ or $n$. Lately, Tomescu [19] characterized all graph of order $n$ having partition dimension $n-2$.

Proposition 1.1. [8] A connected graph $G$ has partition dimension 2 if and only if $G$ is a path.

The following lemma appeared in [8] will be useful to us.

Lemma 1.1. [8] Let $\Pi$ be a resolving partition of $V(G)$ and $u, v \in V(G)$. If $d(u, w)=d(v, w)$ for all $w \in V(G) \backslash\{u, v\}$ then $u$ and $v$ belong to distinct element of $\Pi$.

Next, let $G(V, E)$ be a graph with the vertex-set $V$ and edge-set $E$. Let $c$ be a proper coloring of $G$, namely $c(u) \neq c(v)$ for any adjacent vertices $u$ and $v$ in $G$. Let $c$ be a $k$-coloring of $G$ and $\Pi=\left\{C_{1}, C_{2}, \ldots, C_{k}\right\}$ be a partition of $V(G)$ induced by $c$, where $C_{i}$ is the set of vertices receiving color $i$ for $1 \leq i \leq k$. The color code $c_{\Pi}(v)$ of $v$ is defined as the ordered k-tuple 
$\left(d\left(v, C_{1}\right), d\left(v, C_{2}\right), \ldots, d\left(v, C_{k}\right)\right)$. If all vertices of $G$ have distinct color codes, then $c$ is called a locating-chromatic $k$-coloring of $G$ ( $k$-locating coloring, in short). The locating-chromatic number of a graph $G$, denoted by $\chi_{L}(G)$, is the smallest $k$ such that graph $G$ admits a $k$-locating coloring.

The following theorem taken from paper of Chartrand et al. [6] is a fundamental theorem about the locating chromatic number of a graph.

Theorem 1.1. [6] Let $c$ be a locating coloring in a connected graph $G$. If $u$ and $v$ are distinct vertices of $G$ such that $d(u, w)=d(v, w)$ for all $w \in V(G) \backslash\{u, v\}$, then $c(u) \neq c(v)$. In particular, if $u$ and $v$ are non-adjacent vertices of $G$ such that $N(u)=N(v)$, then $c(u) \neq c(v)$.

Theorem 1.2. [6] The locating chromatic number of a cycle $C_{n}$ is 3 for odd $n$ and 4 for otherwise.

Many papers studied about the partition dimension that containing cycles, see [18, 14, 1]. While, various results of the locating-chromatic number that containing cycles, see [16, 17, 4, 11]. In [3], Asmiati and Baskoro characterized all graph containing cycles with locating-chromatic number three.

In this paper, we show that the locating-chromatic number and the partition dimension of fibonacene graphs are 4 and 3, respectively.

\section{Main Results}

For $h \geq 1$, let $F(h)$ be any fibonacene graph. Then, $F(h)$ has $n=4 h+2$ vertices of which $2 h+4$ are of degree 2 and $2 h-2$ of degree 3 . The $F(h)$ may have type as helicene $\left(H_{h}\right)$, zig-zag fibonacene $\left(Z_{h}\right)$, serpent $\left(S_{h}\right)$, and any combination of them depicted in Figure 1.

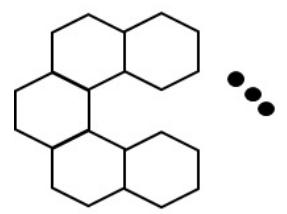

$\mathrm{H}_{\mathrm{h}}$

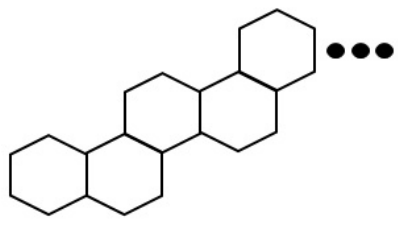

$\mathrm{Z}_{\mathrm{h}}$

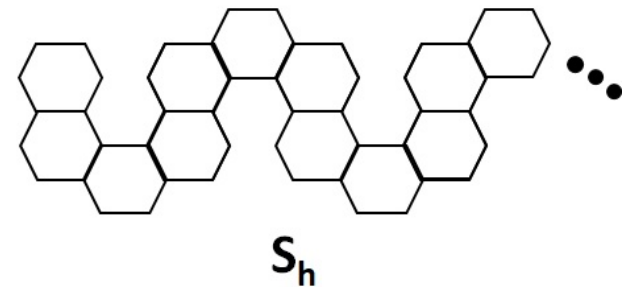

Figure 1. Three extremal fibonacenes.

\subsection{Locating-chromatic number of fibonacene graphs}

This subsection will present the result of the locating-chromatic number for graph $F(h)$.

Theorem 2.1. Let $F(h)$ be a connected fibonacene graph with h hexagons, then $\chi_{L}(F(h))=4$.

Proof. Let $V(F(h))=\left\{v_{0}, v_{1}, v_{2}, \ldots, v_{n-1}\right\}$ where $v_{0}$ is a fixed vertex of degree 2 in terminal hexagon and $v_{0}, v_{1}, v_{2}, \ldots, v_{n-1}$ are cyclicly ordered by Hamiltonian. Let $E(F(h))=\left\{v_{i} v_{i+1} \mid\right.$ $i=0,1, \ldots, n-2\} \cup\left\{v_{0} v_{n-1}\right\} \cup I$, where $I$ is the set of internal edges between two adjacent hexagons. For a contrary suppose $\chi_{L}(F(h)) \leq 3$. Since $F(h)$ is not a path then $\chi_{L}(F(h))>2$. Now, let $c$ be a 3 -coloring on $F(h)$ where $C_{i}=\{v \in V(F(h)) \mid c(v)=i\}$. 
1. There exists one hexagon with 2 colors. let $H$ be the hexagon with 2 colors ( 1 and 2$)$. Now, consider the vertex $u$ with the third color and with minimum distance to hexagon $H$. Without loss of generality, we may assume the vertex $u$ is in the right side of hexagon $H$, depicted in Figure 2. Assume the distance from $u$ to $H$ is equal to $d\left(u, v_{i}\right)$. Then, the color codes of the two neighbors of $v_{i}$ in $H$ are the same, namely $\left(1,0, d\left(u, v_{i}\right)+1\right)$.

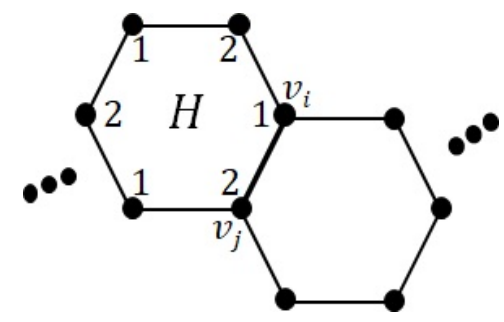

Figure 2. Fibonacene graph coloring with there exists one hexagon with 2 colors.

2. At least one hexagon contains 3 colors.

There are three cases to be considered as depicted in Figure 3. In each case, there are two vertices with the same color codes. These color codes are shown in bold.

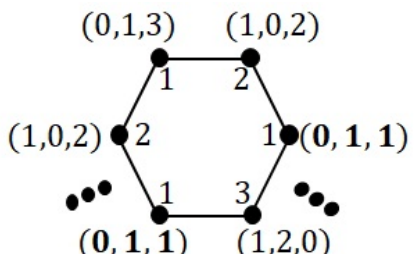

(a)

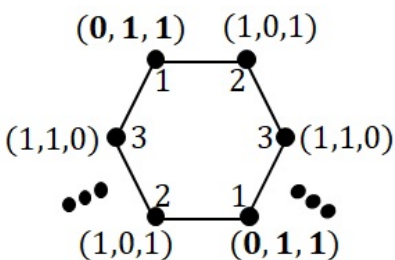

(b)

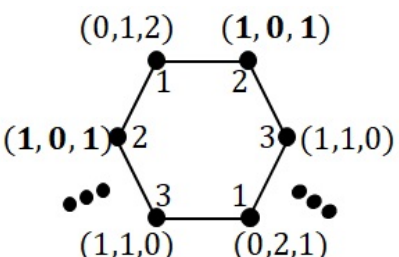

(c)

Figure 3. Fibonacene graph coloring with at least one hexagon contains 3 colors.

Therefore, $\chi_{L}(F(h)) \geq 4$.

Next, define $A_{i}\left(v_{0}\right)=\left\{v \in F(h) \mid d\left(v, v_{0}\right)=i\right\}$ for $i \in\left[0, d_{\max }\right]$ where $d_{\max }=\max$ $\left\{d\left(v, v_{0}\right)\right\}$. For any $i$, we have that $\left|A_{i}\left(v_{0}\right)\right| \leq 4$ since the distribution of all vertices at distance $i$ from $v_{0}$ can be depicted in Figure 4.

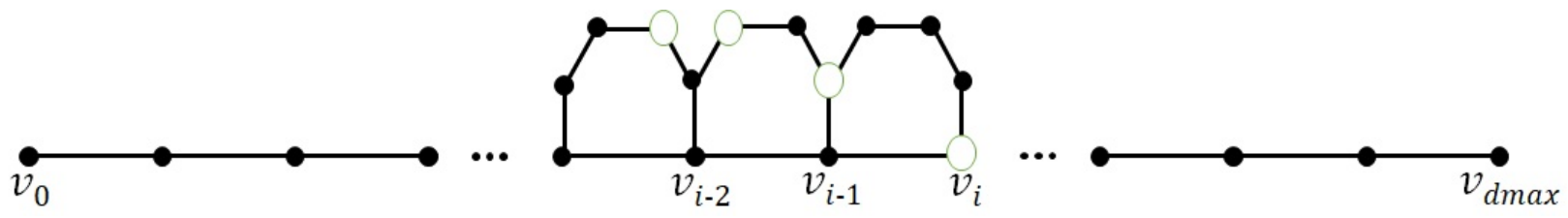

Figure 4. The shortest path from $v_{0}$ to $v_{d_{\max }}$.

Now, define a coloring in $F(h)$ by using algorithm 1 . 
The locating-chromatic number and partition dimension ...

\section{Algorithm 1 (Finding locating coloring in fibonacene graph).}

Let $c: V(F(h)) \rightarrow\{1,2,3,4\}$ such that :

1. $c\left(v_{0}\right)=1$.

2. For $i=1$ to $i_{\max }$ do the following actions.

Case 1. $\left|A_{i}\left(v_{0}\right)\right|=2$ or $\left|A_{i}\left(v_{0}\right)\right|=3$. Define $c\left(A_{i}\left(v_{0}\right)\right) \rightarrow\{2,3,4\}$ such that $\left|c\left(A_{i}\left(v_{0}\right)\right)\right|=$ $\left|A_{i}\left(v_{0}\right)\right|$ and we always use the smallest possible colors in the following ordering.

1. Color a vertex $v$ (if any ) in $A_{i}\left(v_{0}\right)$ where $\left|N(v) \cap A_{i-1}\left(v_{0}\right)\right|=2$.

2. Color at most two vertices $v$ and $w$ in $A_{i}\left(v_{0}\right)$ where $\left|N(v) \cap N(w) \cap A_{i-1}\left(v_{0}\right)\right|=1$.

3. Color a vertex $v$ in $A_{i}\left(v_{0}\right)$ where $\left|N(v) \cap A_{i-1}\left(v_{0}\right)\right|=1$.

Case 2. $\left|A_{i}\left(v_{0}\right)\right|=4$. Let $A_{i}\left(v_{0}\right)=\left\{a_{1}, a_{2}, a_{3}, a_{4}\right\}$. Now define a coloring $c\left(A_{i}\left(v_{0}\right)\right) \rightarrow$ $\{2,3,4\}$ such that we always use the smallest possible colors in the following ordering.

1. Color vertex $a_{1}$ in $A_{i}\left(v_{0}\right)$ where $\left|N\left(a_{1}\right) \cap A_{i-1}\left(v_{0}\right)\right|=2$.

2. Color vertex $a_{2}$ in $A_{i}\left(v_{0}\right)$ and $c\left(a_{2}\right)=c\left(a_{1}\right)$, where $\left|N\left(a_{1}\right) \cap N\left(a_{2}\right) \cap A_{i-1}\left(v_{0}\right)\right|=$ 1.

3. Color a vertex $v \in A_{i+1}\left(v_{0}\right)$ with $v \in N\left(a_{2}\right)$ such that $c(v)=c(s)$ where $s \in$ $N\left(a_{1}\right) \cap N\left(a_{2}\right) \cap A_{i-1}\left(v_{0}\right)$.

4. Color vertex $a_{3}$ where $a_{3} \in N(v) \cap A_{i}\left(v_{0}\right)$.

5. Color the remaining vertex in $A_{i}\left(v_{0}\right)$.

\section{end algorithm 1.}

We will show that $c$ is a locating-coloring on $F(h)$. To do so, we have to prove that for any two vertices $x$ and $y$ in $F(h)$, their color codes are distinct. Let $\Pi=\left\{C_{1}, C_{2}, C_{3}, C_{4}\right\}$ be the partition of $V(F(h))$ induced by the above coloring $c$ with $C_{i}=\{v \in F(h) \mid c(v)=i\}$. Take any two vertices $x$ and $y$ in $F(h)$ such that $c(x)=c(y)$. Of course $x \neq v_{0}$ and $y \neq v_{0}$. If $d\left(x, v_{0}\right) \neq d\left(y, v_{0}\right)$ then $c_{\Pi}(x) \neq c_{\Pi}(y)$. Now, if $d\left(x, v_{0}\right)=d\left(y, v_{0}\right)$ then $x, y \in A_{i}\left(v_{0}\right)$ for some $i$ and $\left|A_{i}\left(v_{0}\right)\right|=4$. So $c_{\Pi}(x) \neq c_{\Pi}(y)$ because $d\left(x, C_{j}\right)=1$ and $d\left(y, C_{j}\right)=2$ for some $j \in\{2,3,4\}$.

Therefore, $c$ is a locating-coloring on $F(h)$ and $\chi_{L}(F(h))=4$.

For $h \geq 1$, let $G(h)$ be any unbranched catacondensed benzenoid graph. In this case, $G(h)$ can have linearly annelated hexagons as depicted in Figure 5. A hexagon is said to be linearly annelated if it is adjacent to exactly two other hexagons and it does not have two adjacent vertices of degree 2 . Therefore, any graph $G(h)$ can be a fibonacene or not.

Theorem 2.2. Let $G(h)$ be a connected unbranched catacondensed benzenoid graph with $h$ hexagons, then $\chi_{L}(G(h))=4$.

Proof. The proof is the same as the one of Theorem 2.1, since the method used in Theorem 2.1 can be also applied to the graph that contains linearly annelated hexagons. 


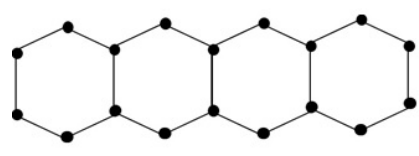

$a$

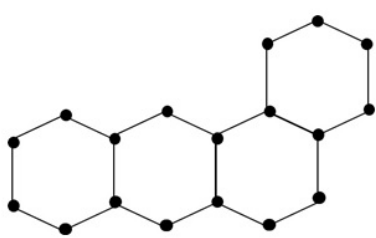

$b$

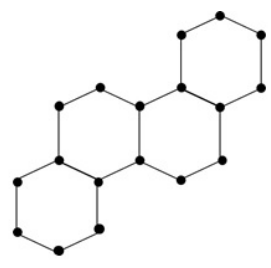

C

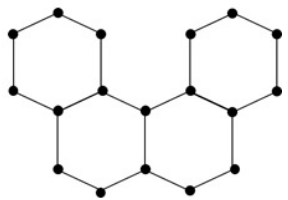

$d$

Figure 5. Unbranched catacondensed benzenoid graphs with 4 hexagons. The graphs in (c) and (d) are fibonacenes and the graphs in (a) and (b) contain linearly annelated hexagons.

\subsubsection{Partition dimension of fibonacene graphs}

This subsection will present the result of the partition dimension for fibonacene graphs.

Theorem 2.3. Let $F(h)$ be a connected fibonacene graph with hexagons, then $p d(F(h))=3$.

Proof. Let $V(F(h))=\left\{v_{0}, v_{1}, v_{2}, \ldots, v_{n-1}\right\}$ where $v_{0}$ is a fixed vertex of degree 2 in terminal hexagon and $v_{0}, v_{1}, v_{2}, \ldots, v_{n-1}$ are cyclicly ordered by Hamiltonian. Let $E(F(h))=\left\{v_{i} v_{i+1} \mid\right.$ $i=0,1, \ldots, n-2\} \cup\left\{v_{0} v_{n-1}\right\} \cup I$, where $I$ is the set of internal edges between two adjacent hexagons. According to Proposition 1.1, $p d(F(h)) \geq 3$. For $i \in\left[0, d_{\max }\right]$ where $d_{\max }=\max$ $\left\{d\left(v, v_{0}\right)\right\}$, define $A_{i}\left(v_{0}\right)=\left\{v \in(F(h)) \mid d\left(v, v_{0}\right)=i\right\}$. Since the distribution of all vertices at distance $i$ from $v_{0}$ can be depicted in Figure 4, then $\left|A_{i}\left(v_{0}\right)\right| \leq 4$ for any $i$.

Next, let $\Pi_{1}=\left\{S_{1}, S_{2}, S_{3}\right\}$ be a partition on $V(F(h))$ induced by a mapping $l: V(F(h)) \longrightarrow$ $\{1,2,3\}$ by using the following algorithm.

\section{Algorithm 2 (Finding resolving partition in fibonacene graph.)}

Partition all vertices in $V(F(h))$ by following steps.

1. Define $l\left(v_{0}\right)=1$ and $l\left(v_{d_{\max }}\right)=2$.

2. For $i=1$ to $d_{\max }-1$ do

Case $1\left|A_{i}\left(v_{0}\right)\right|=2$ and let $A_{i}\left(v_{0}\right)=\left\{a_{1}, a_{2}\right\}$. Then, assign $l\left(a_{1}\right)=2$ and $l\left(a_{2}\right)=3$ if $\left|N\left(a_{1}\right) \cap A_{i-1}\left(v_{0}\right)\right| \geq\left|N\left(a_{2}\right) \cap A_{i-1}\left(v_{0}\right)\right|$.

Case $2\left|A_{i}\left(v_{0}\right)\right|=3$ and let $A_{i}\left(v_{0}\right)=\left\{a_{1}, a_{2}, a_{3}\right\}$. Then, these three vertices $a_{1}, a_{2}, a_{3}$ must be located in two neighboring hexagons. Let $a_{1}$ be the vertex contained in the closest hexagon to terminal hexagon that contains $v_{0}$. Now choose $a_{3}$ such that $N\left(a_{1}\right) \cap N\left(a_{3}\right) \notin A_{i-1}\left(v_{0}\right) \cup A_{i+1}\left(v_{0}\right)$.

1. If $\left|A_{i-1}\left(v_{0}\right)\right|=2$ or $\left|A_{i-1}\left(v_{0}\right)\right|=3$, then $l\left(a_{1}\right)=l\left(a_{3}\right)=2$ and $l\left(a_{2}\right)=3$.

2. If $\left|A_{i-1}\left(v_{0}\right)\right|=4$ and let $k$ be the smallest nonnegative integer such that $\left|A_{i-2-k}\right|=$ 3 , then assign

$$
\left.l\left(a_{1}\right), l\left(a_{2}\right), l\left(a_{3}\right)\right)= \begin{cases}(3,3,2), & \text { if } k \equiv 0(\bmod 4) \\ (2,3,2), & \text { if } k \equiv 1(\bmod 4) \\ (2,2,3), & \text { if } k \equiv 2(\bmod 4) \\ (2,3,2), & \text { if } k \equiv 3(\bmod 4) .\end{cases}
$$


Case $3\left|A_{i}\left(v_{0}\right)\right|=4$ and let $A_{i}\left(v_{0}\right)=\left\{a_{1}, a_{2}, a_{3}, a_{4}\right\}$. Then, these four vertices $a_{1}, a_{2}, a_{3}, a_{4}$ must be located in three hexagons consequtively. Let $a_{1}$ be the vertex contained in the closest hexagon to terminal hexagon that contains $v_{0}$ and choose $a_{2}$ such that $N\left(a_{1}\right) \cap N\left(a_{2}\right) \in A_{i-1}\left(v_{0}\right)$. Let $a_{4}$ be the vertex contained in the farthest hexagon to terminal hexagon that contains $v_{0}$ and choose $a_{3}$ such that $N\left(a_{3}\right) \cap N\left(a_{4}\right) \in A_{i-1}\left(v_{0}\right)$. Moreover $N\left(a_{2}\right) \cap N\left(a_{3}\right) \in A_{i+1}\left(v_{0}\right)$. Let $k$ be the smallest nonnegative integer such that $\left|A_{i-2-k}\right|=3$, then assign

$$
\left(l\left(a_{1}\right), l\left(a_{2}\right), l\left(a_{3}\right), l\left(a_{4}\right)\right)= \begin{cases}(2,3,3,2), & \text { if } k \equiv 0(\bmod 4) \\ (3,3,2,2), & \text { if } k \equiv 1(\bmod 4) \\ (2,2,2,3), & \text { if } k \equiv 2(\bmod 4) \\ (2,2,3,2), & \text { if } k \equiv 3(\bmod 4) .\end{cases}
$$

\section{end algorithm 2.}

We will show that $l$ is a resolving partition on $F(h)$. To do so, we have to show that for any two vertices $x$ and $y$, their repsesentations will be sdistinct. Take any two vertices $x$ and $y$ in $F(h)$ such that $l(x)=l(y)$. Of course, $x \neq v_{0}$ and $y \neq v_{0}$. If $d\left(x, v_{0}\right) \neq d\left(y, v_{0}\right)$ then $r\left(x \mid \Pi_{1}\right) \neq r\left(y \mid \Pi_{1}\right)$. Now, if $d\left(x, v_{0}\right)=d\left(y, v_{0}\right)$ then we have $x, y \in A_{i}\left(v_{0}\right)$ for some $i$. Note that the symbol $(a ; b ; \ldots, c)$ represents $\left|A_{i-1}\left(v_{0}\right)\right|=a,\left|A_{i}\left(v_{0}\right)\right|=b$ and $\left|A_{i+k}\left(v_{0}\right)\right|=c$ for some $k$.

1. If $\left|A_{i}\left(v_{0}\right)\right|=3$ then the only possible distribution of the vertices at distance $i-1, i$ and $i+1$ from $v_{0}$ are $(2 ; 3 ; 3),(2 ; 3 ; 4),(3 ; 3 ; 1),(3 ; 3 ; 2),(4 ; 3 ; 1),(4 ; 3 ; 2)$. For the first four cases namely $(2 ; 3 ; 3),(2 ; 3 ; 4),(3 ; 3 ; 1)$, and $(3 ; 3 ; 2)$, all vertices in $A_{i-1}\left(v_{0}\right), A_{i}\left(v_{0}\right)$ and $A_{i+1}\left(v_{0}\right)$ will be partitioned according to Algorithm 2. Therefore, we have $l\left(a_{1}\right)=l\left(a_{3}\right)=2$ and $l\left(a_{2}\right)=3$ so $x=a_{1}$ and $y=a_{3}$. In these case, we have that $r\left(x \mid \Pi_{1}\right) \neq r\left(y \mid \Pi_{1}\right)$, as shown in Figure 6. All vertices in $A_{i-1}\left(v_{0}\right), A_{i}\left(v_{0}\right), A_{i+1}\left(v_{0}\right)$ respectively is indicated by $\square$, $\circ, \triangle$. For the cases of $(4 ; 3 ; 1)$ and $(4 ; 3 ; 2)$, we will discuss later in case of $\left|A_{i}\left(v_{0}\right)\right|=4$.

2. If $\left|A_{i}\left(v_{0}\right)\right|=4$ then the only possible distribution of vertices at distance $i-1, i$ and $i+1$ from $v_{0}$ are $(3 ; 4 ; 3),(3 ; 4 ; 4),(4 ; 4 ; 3),(4 ; 4 ; 4)$. All four cases will be combined with cases of $(4 ; 3 ; 1)$ and $(4 ; 3 ; 2)$. If hexagon $H$ is terminal hexagon then it will get the case of $(4 ; 3 ; 1)$. If hexagon $H$ is not terminal hexagon then there are two possibilities for the addition of hexagon to keep forming a fibonacene graph. If $e_{2}$ is the internal edge then the next image will be obtained. If $e_{1}$ is the internal edge then it will get the case of $(4 ; 3 ; 2)$. Therefore, vertices $x$ and $y$ will be contained in $\circ, \Delta, \nabla, \square, \triangleright$, and $\triangleleft$. All vertices in $A\left(v_{0}\right)$ will be partitioned according to cases 2.2 and 3 Algorithm 2. So, if $x, y \in A_{i}\left(v_{0}\right)$ for some i then $r\left(x \mid \Pi_{1}\right) \neq r\left(y \mid \Pi_{1}\right)$, as shown in Figure 7 and the representations of vertices can see on table 1.

Therefore, since for each vertices on $F(h)$ have distinct representations so $p d(F(h)) \leq 3$. We get $p d(F(h))=3$. 


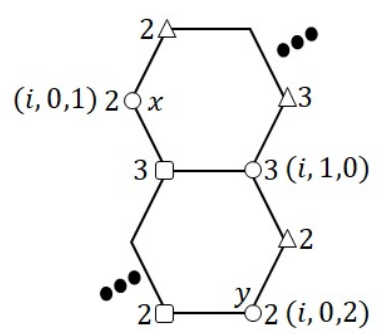

$(2 ; 3 ; 3)$

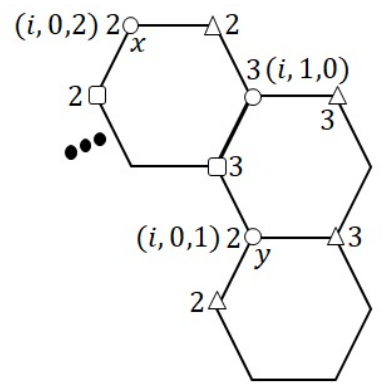

$(2 ; 3 ; 4)$

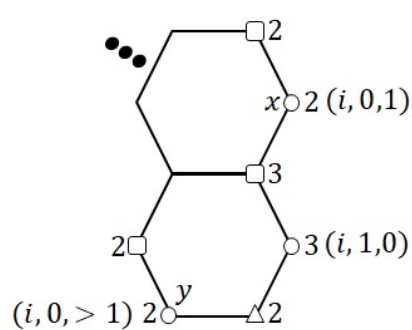

$(3 ; 3 ; 1)$

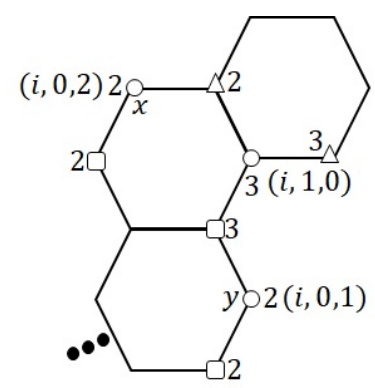

$(3 ; 3 ; 2)$

Figure 6 . The representations of vertices in $\left|A_{i}\left(v_{0}\right)\right|=3$ for the cases of $(2 ; 3 ; 3),(2 ; 3 ; 4),(3 ; 3 ; 1),(3 ; 3 ; 2)$.

\section{Acknowledgement}

This research has been supported by the Research Grant: "Penelitian Dasar Unggulan Perguruan Tinggi (PDUPT)", the Ministry of Research, Technology and Higher Education and the Research Grant "Program Penelitian dan Pengabdian kepada Masyarakat (P3MI), Institut Teknologi Bandung", Indonesia.

\section{References}

[1] Amrullah, E. T. Baskoro, and R. Simajuntak, The partition dimension of a subdivision of a complete graph, Procedia Comput. Sci. 74 (2015), 53-59.

[2] P. G. Anderson, Fibonaccene, in: A. N. Philippou, G. E. Bergum, and A. F. Horadam (Eds.), Fibonacci numbers and their applications, Reidel, Dordrecht, (1986), pp. 1-8.

[3] Asmiati and E. T. Baskoro, Characterizing all graphs containing cycle with the locatingchromatic number 3, AIP Conf. Proc. 1450 (2012), 351-357.

[4] Asmiati, Wamiliana, Devriyadi, and R. Yulianti, On some Petersen graphs having locatingchromatic number four or five, Far East J. Math. Sci. 102 (4) (2017), 769-778.

[5] A. T. Balaban, Chemical graphs. 50. Symmetry and enumeration of fibonacenes (Unbranched catacondensed benzenoids isoarithmic with helicenes and zigzag catafusenes), MATCH Commun. Math. Comput. Chem. 24 (1989), 29-38.

[6] G. Chartrand, D. Erwin, M. A. Henning, P. J. Slater, and P. Zhang, The locating-chromatic number of a graph, Bull. Inst. Combin. Appl. 36 (2002), 89-101.

[7] G. Chartrand, E. Salehi, and P. Zhang, On the partition dimension of a graph, Congr. Numer. 130 (1998), 157-168.

[8] G. Chartrand, E. Salehi, and P. Zhang, The partition dimension of a graph, Aequationes Math. 59 (2000), 45-54. 


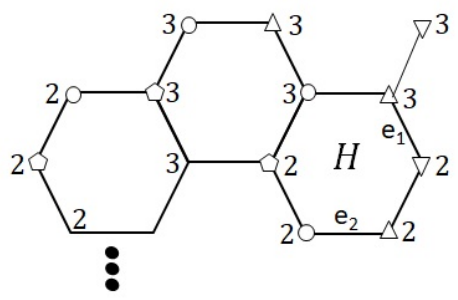

(a) $3 ; 4 ; 3 ; 1(2)$

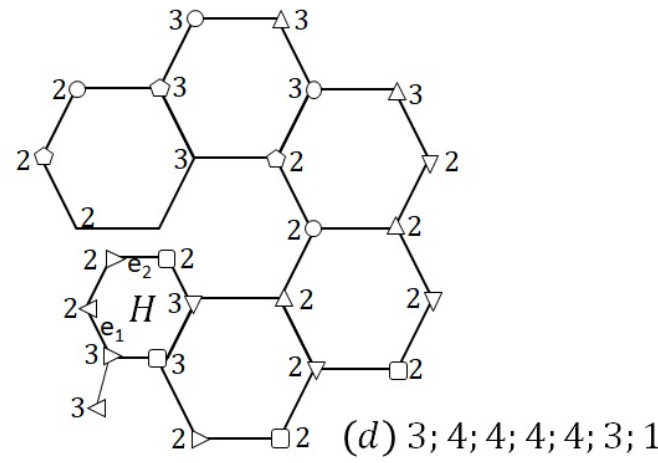

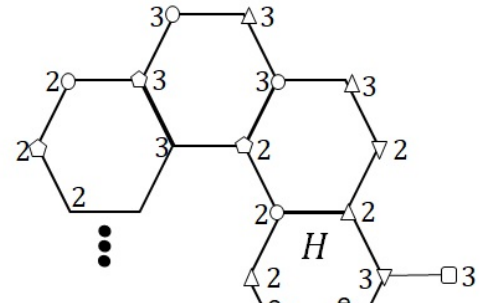

(b) $3 ; 4 ; 4 ; 3 ; 1(2)$

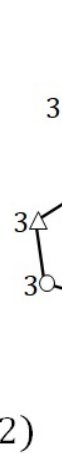

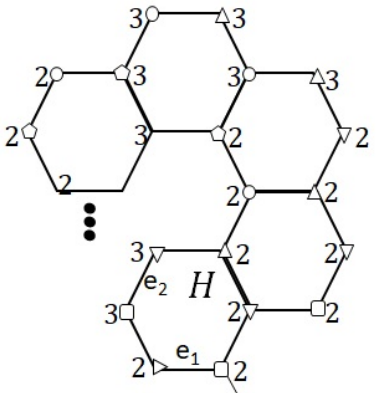

$\triangleright 3$

Figure 7. The cases of $\left|A_{i}\left(v_{0}\right)\right|=4$.

[9] A. A. Dobrynin, I. Gutman, S. Klavžar, and P. Žigert, Wiener index of hexagonal systems, Acta Appl. Math. 72 (2002), 247-294.

[10] T. Došlić and I. Zubac, Saturation number of benzenoid graphs, MATCH Commun. Math. Comput. Chem. 73 (2015), 491-500.

[11] M. Ghanem, H. Al-Ezah, and A. Dabbour, Locating chromatic number of powers of paths and cycles, Symmetry 11 (2019), 389-396.

[12] I. Gutman and S. Klavžar, Chemical graph theory of fibonacenes, MATCH Commun. Math. Comput. Chem. 55 (2006), 39-54.

[13] F. Harary and R. A. Melter, On the metric dimension of a graph, Ars Combin. 2 (1976), 191-195.

[14] I. Javaid, N. K. Raja, M. Salman, and M. N. Azhar, The partition dimension of circulant graphs, World Appl. Sci. J. 18 (2012), 1705-1717.

[15] R. Pepper, An upper bound on the independence number of benzenoid systems, Discrete Appl. Math. 156 (2008), 607-619.

[16] I. A. Purwasih and E. T. Baskoro, The locating-chromatic number for Halin graphs, AIP Conf. Proc. 1450 (2012), 342-345. 
The locating-chromatic number and partition dimension ...

Table 1. Representations of vertices on some cases with $\left|A_{i}\left(v_{0}\right)\right|=4$ and the cases of $(4 ; 3 ; 1)$ and $(4 ; 3 ; 2)$.

\begin{tabular}{|c|c|c|c|c|c|}
\hline- & $a$ & $b$ & $c$ & $d$ & $e$ \\
\hline \multirow{4}{*}{ O } & $(i, 0,1)$ & $(i, 0,1)$ & $(i, 0,1)$ & $(i, 0,1)$ & $(i, 0,1)$ \\
\hline & $(i, 2,0)$ & $(i, 2,0)$ & $(i, 2,0)$ & $(i, 2,0)$ & $(i, 2,0)$ \\
\hline & $(i, 1,0)$ & $(i, 1,0)$ & $(i, 1,0)$ & $(i, 1,0)$ & $(i, 1,0)$ \\
\hline & $(i, 0,2)$ & $(i, 0,2)$ & $(i, 0,2)$ & $(i, 0,2)$ & $(i, 0,2)$ \\
\hline \multirow{4}{*}{$\triangle$} & $(i+1,2,0)$ & $(i+1,2,0)$ & $(i+1,2,0)$ & $(i+1,2,0)$ & $(i+1,2,0)$ \\
\hline & $(i+1,1,0)$ & $(i+1,1,0)$ & $(i+1,1,0)$ & $(i+1,1,0)$ & $(i+1,1,0)$ \\
\hline & $(i+1,0,2)$ & $(i+1,0,1)$ & $(i+1,0,2)$ & $(i+1,0,2)$ & $(i+1,0,2)$ \\
\hline & - & $(i+1,0,3)$ & $(i+1,0,1)$ & $(i+1,0,1)$ & $(i+1,0,1)$ \\
\hline \multirow{4}{*}{$\nabla$} & - & $(i+2,0,1)$ & $(i+2,0,1)$ & $(i+2,0,1)$ & $(i+2,0,1)$ \\
\hline & - & $(i+2,1,0)$ & $(i+2,0,3)$ & $(i+2,0,3)$ & $(i+2,0,3)$ \\
\hline & - & $(i+2,0,2)$ & $(i+2,0,2)$ & $(i+2,0,2)$ & $(i+2,0,2)$ \\
\hline & - & - & $(i+2,1,0)$ & $(i+2,1,0)$ & $(i+2,1,0)$ \\
\hline \multirow{4}{*}{$\square$} & - & - & $(i+3,0,3)$ & $(i+3,0,3)$ & $(i+3,0,3)$ \\
\hline & - & - & $(i+3,0,2(1))$ & $(i+3,0,2)$ & $(i+3,0,2)$ \\
\hline & - & - & $(i+3,1,0)$ & $(i+3,1,0)$ & $(i+3,1,0)$ \\
\hline & - & - & - & $(i+3,0,1)$ & $(i+3,0,1)$ \\
\hline \multirow{4}{*}{$\triangleright$} & - & - & - & $(i+4,0,1)$ & $(i+4,0,1)$ \\
\hline & - & - & - & $(i+4,1,0)$ & $(i+4,2,0)$ \\
\hline & - & - & - & $(i+4,0,2)$ & $(i+4,1,0)$ \\
\hline & - & - & - & - & $(i+4,0,2)$ \\
\hline \multirow{4}{*}{$\triangleleft$} & - & - & - & - & $(i+5,2,0)$ \\
\hline & - & - & - & - & $(i+5,1,0)$ \\
\hline & - & - & - & - & $(i+5,0,2)$ \\
\hline & - & - & - & - & - \\
\hline
\end{tabular}

[17] I. A. Purwasih, E. T. Baskoro, H. Assiyatun, and W. Djohan, The locating-chromatic number for a subdivision of a wheel on one cycle edge, AKCE Int. J. Graphs Combin. 10 (3) (2013), 327-336.

[18] I. Tomescu, I. Javaid, and Slamin, On the partition dimension and connected partition dimension of wheels, Ars. Combin. 84 (2007), 311-317.

[19] I. Tomescu, Discrepancies between metric dimension and partition dimension of a connected graph, Discrete Math. 308 (2008), 5026-5031. 